\title{
A REVIEW OF TECHNIQUES FOR PARAMETER SENSITIVITY ANALYSIS OF ENVIRONMENTAL MODELS
}

\author{
D. M. HAMBY* \\ Westinghouse Savannah River Company Savannah River Technology Center Aiken, SC 29808, \\ U.S.A.
}

\begin{abstract}
Mathematical models are utilized to approximate various highly complex engineering, physical, environmental, social, and economic phenomena. Model parameters exerting the most influence on model results are identified through a 'sensitivity analysis'. A comprehensive review is presented of more than a dozen sensitivity analysis methods. This review is intended for those not intimately familiar with statistics or the techniques utilized for sensitivity analysis of computer models. The most fundamental of sensitivity techniques utilizes partial differentiation whereas the simplest approach requires varying parameter values one-at-a-time. Correlation analysis is used to determine relationships between independent and dependent variables. Regression analysis provides the most comprehensive sensitivity measure and is commonly utilized to build response surfaces that approximate complex models.
\end{abstract}

\section{Introduction}

Mathematical models are utilized to approximate various highly complex engineering, physical, environmental, social, and economic phenomena. Model development consists of several logical steps, one of which is the determination of parameters which are most influential on model results. A 'sensitivity analysis' of these parameters is not only critical to model validation but also serves to guide future research efforts.

Modelers may conduct sensitivity analyses for a number of reasons including the need to determine: (1) which parameters require additional research for strengthening the knowledge base, thereby reducing output uncertainty; (2) which parameters are insignificant and can be eliminated from the final model; (3) which inputs contribute most to output variability; (4) which parameters are most highly correlated with the output; and (5) once the model is in production use, what consequence results from changing a given input parameter. There are many different ways of conducting sensitivity analyses; however, in answering these questions the various analyses may not produce identical results (Iman and Helton, 1988).

Generally, sensitivity analyses are conducted by: (a) defining the model and its independent and dependent variables (b) assigning probability density functions to each input parameter, (c) generating an input matrix through an appropriate random sampling method, calculating an output vector, and (e) assessing the influences and

* Current address: 2505 School of Public Health-I, University of Michigan, Ann Arbor, MI 48109-2029, U.S.A. 
relative importance of each input/output relationship (Iman et al. 1981a; Iman et al., 1981b; Helton and Iman, 1982; Helton et al., 1985; Helton et al., 1986).

The literature contains details on the types of sensitivity analyses utilized for various modeling situations. More than a dozen methods have been reviewed (Bellman and Astrom, 1970; Cukier et al., 1973; Morisawa and Inoue, 1974; Krieger et al., 1977; Box et al., 1978; Oblow, 1978; Conover, 1980; Gardner et al., 1980; Demiralp and Rabitz, 1981; Gardner et al., 1981; Iman et al., 1981b; Hoffman and Gardner, 1983; Downing et al., 1985; Helton et al., 1985; Crick et al., 1987; IAEA, 1989; Iman and Helton, 1991; Hamby, 1993). This paper is a comprehensive assessment of several of the more practical methods for conducting parameter sensitivity studies, ranging from one-at-a-time sensitivity measures to standardized regression coefficients to statistical tests based on partitioning of empirical input distributions. Generally, method practicality is determined based on the calculational ease and the usefulness of results.

Conceptual descriptions of methods are given so that investigators conducting sensitivity analyses can judge the relative merits weighted against the expense, resource drain, and usefulness of each technique. Discussions of the following sensitivity analysis techniques are included: differential analysis, one-at-a-time design, factorial design, the derivation of sensitivity and importance indices, subjective analysis, construction of scatter plots, the relative deviation method, relative deviation ratios, correlation coefficients, rank transformation, rank correlation coefficients, partial correlation coefficients, regression techniques, standardized regression techniques, the Smirnov test statistic, the Cramer-von Mises test, MannWhitney test, and the squared ranks test. A separate paper (Hamby, 1995) applies each of these tests in a numerical comparison of parameter sensitivity methods on a probabilistic dose assessment methodology (Hamby, 1993).

A few of the sensitivity analysis techniques currently in the literature are intended for highly complex or very large models and are discussed below only briefly. These include structural identifiability (Bellman and Astrom, 1970) and methods using adjoint equations (Oblow, 1978), Fourier analysis (Cukier et al. 1973; Helton et al. 1991), and Green's functions (Demiralp and Rabitz, 1981). Structural identifiability was introduced by Bellman and Astrom (1970) as a tool in biological modeling to assess the internal structure of a system from input-output measurements (Cobelli and Romanin-Jacur, 1976; Cobelli and DiStefano, 1980). The adjoint method has been demonstrated by several investigators in the fields of climatological modeling, reactor thermal hydraulics, and reactor safety (Koda et al. 1979; Cacuci et al., 1980; 1983; Hall et al., 1982). In this approach, 'exact' (Downing et al. 1985) sensitivities are calculated as defined by partial derivatives. The Fourier amplitude sensitivity test (FAST) (Cukier et al. 1973, 1978) has been used to solve coupled nonlinear rate equations by Schaibly and Shuler (1973) and Cukier et al., (1975). FAST has also been used by Dickinson and Gelinas (1976) to study sensitivities of parameters used to model atmospheric chemical kinetics, by Koda et al. (1979) to study atmospheric diffusion, and several others (Leipmann 
and Stephanopoulos, 1985; Kim et al., 1988). Theoretical aspects of a method using Green's functions are presented by Demiralp and Rabitz (1981). In this method, Green's functions are used to avoid solving the differential equations for each model parameter each time the base-case parameter values are changed (Hall et al., 1982). Iman and Helton (1985) briefly discuss and apply all three methods.

\section{Sensitivity Analysis Methods}

Many authors, when referring to the degree to which an input parameter affects the model output, use the terms 'sensitive', 'important', 'most influential', 'major contributor', 'effective', or 'correlated' interchangeably (Krieger et al., 1977; Downing et al. 1985; Iman, 1987; Iman and Helton, 1988; Marguiles et al., 1991). Crick et al. (1987) have made a distinction by referring to 'important' parameters as those whose uncertainty contributes substantially to the uncertainty in assessment results, and 'sensitive' parameters as those which have a significant influence on assessment results. The consensus among authors is that models are indeed sensitive to input parameters in two distinct ways: (1) the variability, or uncertainty, associated with a sensitive input parameter is propagated through the model resulting in a large contribution to the overall output variability, and (2) model results can be highly correlated with an input parameter so that small changes in the input value result in significant changes in the output.

The necessary distinction between important and sensitive parameters is in the type of analysis being conducted: uncertainty analysis (parameter importance) or sensitivity analysis (parameter sensitivity). An important parameter is always sensitive because parameter variability will not appear in the output unless the model is sensitive to the input. A sensitive parameter, however, is not necessarily important because it may be known precisely, thereby having little variability to add to the output.

At the completion of an analysis on parameter sensitivity the analyst holds a list, or 'sensitivity ranking', of the input parameters sorted by the amount of influence each has on the model output. More than a dozen sensitivity techniques are described, each of which would result in a slightly different sensitivity ranking. The actual ranking is not as important as is the specification of which parameters consistently appear near the top of the list. Disagreement among rankings by the various methods for variables of lesser importance is not of practical concern since these variables have little or no influence on model output.

Throughout this paper a generalized model is utilized that contains several independent variables, $X=\left(X_{1}, \ldots, X_{n}\right)$, and one dependent variable $Y$, where $Y=f(X)$. These methods can also be applied to models with several dependent variables. Sensitivity methods will be addressed in three groups:

(1) those that operate on one variable at a time; 
(2) those that rely on the generation of an input matrix and an associated output vector;

(3) those that require a partitioning of a particular input vector based on the resulting output vector.

\subsection{Differential SEnsitivity AnALysis}

Differential analysis, also referred to as the direct method, is discussed first since it is the backbone of nearly all other sensitivity analysis techniques. Methods in the literature range from solving simple partial derivatives to spatial and temporal sensitivity analyses (Morisawa and Inoue, 1974; Atherton et al., 1975; Dickinson and Gelinas, 1976; Koda et al., 1979; Dunker, 1981; Gardner et al., 1981; Summers and McKellar, 1981; Koda, 1982; Helton et al., 1985; Iman and Helton, 1985; Helton et al., 1986; Iman and Helton, 1988; Helton et al., 1991; Zimmerman et al., 1991).

A sensitivity coefficient is basically the ratio of the change in output to the change in input while all other parameters remain constant (Krieger et al., 1977). The model result while all parameters are held constant is defined as the 'base case'. Differential techniques are structured on the behavior of the model given a specific set of parameter values, e.g. assuming the base-case scenario is with all parameter values set to their mean. Morisawa and Inoue (1974) use the differential method as a means of selecting desirable conditions for underground waste disposal sites in Japan. They note, however, that, with the direct method, the magnitude of variable sensitivity is dependent on the base-case scenario. A major drawback is that this localized behavior may not be applicable for realms far from the base case.

Differential analysis of parameter sensitivity is based on partial differentiation of the model in aggregated form. It can be thought of as the propagation of uncertainties (Cunningham et al., 1980). A first-order Taylor series approximation is applied to the dependent variable, $y$, as a function of the independent variables $X=\left(x_{1}, \ldots, X_{n}\right)$. The variance of $Y, V(Y)$, is calculated using the general error propagation formula, i.e.,

$$
V(Y)=\sum_{i=1}^{n}\left(\frac{\partial Y}{\partial X_{i}}\right)^{2} V\left(X_{i}\right) .
$$

The variance in $Y$ is utilized as a measure of uncertainty in model predictions while the variance in $X_{i}$, weighted by the first-order partial of $Y$ with respect to $X_{i}$, provides a measure of model sensitivity to $X_{i}$ (Helton et al., 1985). This method is a linearized theory and is valid only for small parameter uncertainties (Koda et al., 1979). A statistical sensitivity analysis consists of computing the variance and the expected value of each model output and ranking the contributions to the variance (Atherton et al., 1975). Iman (1987) uses a matrix-based approach to solving the partial derivatives since matrix notation is efficient and because it allows for the analysis of large problems. Several recent investigations have utilized the 
GRESS (Gradient Enhanced Software System) computer code, developed at Oak Ridge National Laboratory (Worley and Horwedel, 1986; Horwedel et al., 1988), to calculate sensitivity coefficients using the direct method (Helton et al., 1991; Yu et al., 1991; Zimmerman et al., 1991).

Sensitivity analyses using partial differentiation techniques are computationally efficient (Helton et al., 1985); however, the effort required in solving these equations can be quite intensive. Derivatives of simple functions may be generated by less rigorous analytical or differencing schemes, but more complicated models often require complex numerical procedures (Iman and Helton, 1988). When an explicit algebraic equation describes the relationship between the independent variables and the dependent variable, the sensitivity analysis is easy to perform (Atherton et al., 1975). In this case, the sensitivity coefficient, $\phi_{i}$, for a particular independent variable can be calculated from the partial derivative of the dependent variable with respect to the independent variable, i.e.

$$
\phi_{i}=\frac{\partial Y}{\partial X_{i}}\left(\frac{X_{i}}{Y}\right)
$$

where the quotient, $X_{i} / Y$, is introduced to normalize the coefficient by removing the affects of units. Inherent to this calculation are the assumptions that the higher ordered partials are negligible and there is no correlation between input parameters (Atherton et al., 1975; Gardner et al., 1981). For large sets of equations, the partial derivative can be approximated as a finite difference and output values calculated for small changes in the input parameter (Downing et al., 1985). Thus, if nonlinearities are neglected, the partial derivative can be approximated as,

$$
\phi_{i}=\frac{\% \Delta Y}{\% \Delta X_{i}} .
$$

Gardner et al. (1981) report that the partial derivative approach to sensitivity analysis may become so complex that implementation is impractical. Also, since this approach is only valid for small changes in parameter values, when parameter variability is allowed to take on 'realistic' values, the direct approach is seriously violated (Gardner et al., 1981). The differential analysis is typically much more demanding to implement than other sensitivity methods and yet provides only comparable results.

\subsection{ONE-AT-A-Time SENSITIVITY MEAsuRes}

Conceptually, the simplest method to sensitivity analysis is to repeatedly vary one parameter at a time while holding the others fixed (Gardner et al., 1980; O'Neill et al., 1980; Downing et al., 1985; Breshears, 1987; Crick et al., 1987; Yu et al., 1991). A sensitivity ranking can be obtained quickly by increasing each parameter by a given percentage while leaving all others constant, and quantifying the change in model output. This type of analysis has been referred to as a 'local' sensitivity 
analysis (Crick et al., 1987) since it only addresses sensitivity relative to the point estimates chosen and not for the entire parameter distribution. The RESRAD code, developed at Argonne National Laboratory for the Department of Energy, calculates dose (or risk) to humans from residual soil contamination (Yu et al., 1991). The package contains a routine that uses the local sensitivity method by varying user-specified parameter values and printing a graphical representation of parameter sensitivity over time.

A more powerful test of local sensitivity examines the change in output as each parameter is individually increased by a factor of its standard deviation. This sensitivity measure takes into account the parameter's variability and the associated influence on model output. Downing et al. (1985) evaluate parameters at their mean value and then vary them by \pm 4 standard deviations to measure the effect on the output.

\subsection{FACTORIAL DESIGN}

A factorial analysis involves choosing a given number of samples for each parameter and running the model for all combinations of the samples (Box et al., 1978; Rose, 1983). The results obtained in this fashion are then utilized to estimate parameter sensitivity. For example, a model has five parameters and it is determined, rather arbitrarily, that each parameter will be sampled at three specific locations, e.g. the 25 th, 50th, and 75 th percentiles. This experimental design would require $5^{3}=125$ model runs. It is evident from this example that a large number of parameters quickly prohibits a thorough examination of the model because of the tremendous number of model runs required. In some cases, the same information can be obtained from a reduced number of trials, using a fractional factorial design (Box et al., 1978).

\subsection{THE SENSITIVITY INDEX}

Another of the simple methods of determining parameter sensitivity is to calculate the output \% difference when varying one input parameter from its minimum value to its maximum value (Hoffman and Gardner, 1983; Bauer and Hamby, 1991). Hoffman and Gardner (1983) advocate utilizing each parameter's entire range of possible values in order to assess true parameter sensitivities. The 'sensitivity index' (SI) is calculated using,

$$
S I=\frac{D_{\max }-D_{\min }}{D_{\max }},
$$

where $D_{\min }$ and $D_{\max }$ represent the minimum and maximum output values, respectively, resulting from varying the input over its entire range (Hoffman and Gardner, 1983). This figure-of-merit provides a good indication of parameter and model variability. 


\subsection{IMPORTANCE FACTORS}

Downing et al. (1985) have introduced three importance factors. Their measures are calculated from data collected after a five-point one-at-a-time analysis; model output is recorded for each parameter at its mean value, \pm 2 standard deviations, and \pm 4 standard deviations. The first importance factor is defined as parameter uncertainty (defined as two standard deviations of the input) multiplied by parameter sensitivity (defined as the change in output divided by change in input). The second is the positive difference in the maximum output value and the minimum output value. And, third, they estimate importance utilizing the output sample variance.

\subsection{SubJeCtive SENSITIVITY ANALYSIS}

The final sensitivity method based on analysis of individual parameters is the subjective method (Downing et al., 1985). The method is rather simple and only qualitative since it relies on the opinions of experienced investigators to determine, a priori, which parameters can be discarded due to lack of influence on model results. One advantage is that, for large models, where most other methods are impractical, the subjective method can be used as a first cut to reduce the number of input parameters to a manageable size.

\section{Parameter Sensitivity Analysis Utilizing Random Sampling Methods}

To this point, sensitivity has been assessed on individual parameters without regard to the combined variability resulting from considering all input parameters simultaneously. Random sampling (e.g. simple random sampling, Monte Carlo, Latin hypercube, etc.) of input parameters generates input and output distributions useful in assessing model and parameter uncertainties in a 'global' sense (McKay et al., 1979; Iman and Conover, 1980; Iman et al., 1981a; Helton and Iman, 1982; Iman and Conover, 1982; Iman and Shortencarier, 1984; Reed et al., 1984; Downing et al., 1985; Kim et al., 1988; Iman, 1987; Stein, 1987; Helton et al., 1991). Gardner et al. (1980) refer to parameter sensitivity studies of this type as 'parameter error analysis'.

A large array of randomly selected input parameter values and calculated output values provides a means for determining parameter sensitivity through a variety of procedures. The influence of other input parameters is meaningful to consider in uncertainty and sensitivity analyses since overall model performance is of importance. Distribution effects are meaningful because parameter sensitivity depends, not only on the range and distribution of an individual input parameter, but also on those of other parameters to which the model is sensitive (Iman et al., 1981a). Parameter sensitivity is dependent on the interactions and influences of all parameters. 


\subsection{SCATTER PLOTS}

Parameter sensitivity can be determined qualitatively by plots of input vs. output values, or quantitatively by calculations of correlation coefficients or regression analysis. Scatter plots of input vs. output are useful for quick determinations of the degree of correlation and the linearity of the input/output relationship (Helton et al., 1986; Crick et al., 1987; Iman and Helton, 1988; Helton et al., 1991; Helton et al., 1993). They may also reveal unexpected relationships between input and output variables that can provide insight as to how other investigations (e.g. regression analysis) might be performed.

\subsection{THE IMPORTANCE INDEX}

Hoffman and Gardner (1983) have also introduced an 'importance index', $I_{i}$, which is equal to the variance of the parameter value, $s_{X}^{2}$, divided by the variance of the dependent values, $s_{Y}^{2}$, i.e.

$$
I_{i}=\frac{s_{X_{i}}^{2}}{s_{Y}^{2}}
$$

where $s$ refers to the variance of the raw data for additive models and to the variance of the log-transformed data for multiplicative models. This measure of importance is based on the parameter's fractional contribution to total variability, or uncertainty. Variable importance is estimated by Cunningham et al. (1980) through the use of a combination of the fractional contribution to output variability and the resulting change in output given individual change in input.

\subsection{The 'Relative Deviation' Method}

One sensitivity ranking method utilizes random sampling techniques and measures the amount of variability introduced into the model output while varying each input parameter, one-at-a-time, according to its probability density function. This method is similar to the local sensitivity method with the exception that a much larger sampling is made of the input distribution. The sensitivity figure-of-merit is the 'relative deviation' (RD), the ratio of the standard deviation to the mean of the output density function (Hamby, 1993), and is similar to the coefficient of variation (standard deviation $\times 100 /$ mean). This test provides an indication of each parameter's contribution to the variability present in the model output and, to a degree, the extent of correlation that exists between the input and output variable.

\subsection{The 'Relative Deviation Ratio'}

Given two input distributions, one narrow and one wide, producing identical output distributions, a model will be more sensitive to the input parameter of the narrow 
distribution. Accordingly, this test statistic will be the ratio of the output distribution's relative deviation to the input distribution's relative deviation and is similar to the importance index proposed by Hoffman and Gardner (1983). A large value of this 'relative deviation ratio' (RDR) indicates that either the output distribution varies widely or that the input distribution is relatively narrow. Additionally, information on the amount of variability added to the total output variability by the model itself is gained from this statistic. A value greater than unity indicates that uncertainty propagated through the model is being increased due to the model's structure and its high sensitivity to that particular variable. An RDR of 1 indicates that all input uncertainty is passed through the model and appears as output uncertainty, while a value less than unity indicates that the model is less sensitive to the parameter, thereby contributing to output uncertainty to a lesser degree.

\subsection{PEARSON'S $r$.}

A quantitative estimate of linear correlation can be determined by calculating a simple correlation coefficient on the parameter values of input and output. Gardner et al. (1981) recommend using simple correlation coefficients, derived from Monte Carlo simulations, as a reasonable way to rank model parameters according to their contribution to prediction uncertainty. Pearson's product moment correlation coefficient is denoted by $r$ and is defined as

$$
r=\frac{\sum_{j=1}^{n}\left(X_{i j}-\bar{X}_{i}\right)\left(Y_{j}-\bar{Y}\right)}{\left[\sum_{j=1}^{n}\left(X_{i j}-\bar{X}_{i}^{2} \sum_{j=1}^{n}\left(Y_{j}-\bar{Y}\right)^{2}\right]^{1 / 2}\right.}
$$

for the correlation between $X_{i}$ and $Y$ (Conover, 1980). The larger the absolute value of $r$ the stronger the degree of linear relationship between the input and output values (IAEA 1989). A negative value of $r$ indicates the output is inversely related to the input. A linear regression on the data can be used to determine the correlation coefficient from the square root of the coefficient of determination, $R^{2}$.

Major drawbacks of utilizing the correlation coefficient for sensitivity ranking include the inherent assumption that the input/output relationship is linear and the possibility that input parameters strongly correlated to one another may result in apparent input/output correlations (Hoffman and Gardner, 1983; Crick et al., 1987; IAEA, 1989). In addition, a large number of trials may prohibit hand calculations of the correlation coefficient.

\subsection{THE RANK TRANSFORMATION}

One of the problems encountered in calculating test statistics, e.g. correlation coefficients, from raw data is that the data are not necessarily linear. A method of reducing the effects of nonlinear data is to use the rank transformation (Iman and 
Conover, 1979). The transformation of raw data into ranks has been shown to work quite well if the dependent variable is a monotonic function of the independent variables (Iman and Conover, 1979). Rank transformation linearizes monotonic nonlinear relationships between variables and reduces the effects of extreme values (Helton and Iman, 1982). This transformation converts the sensitivity measure from one of linearity to one of monotonicity.

\subsection{SPEARMAN'S $\rho$}

If the input/output associations are monotonic then rank transformations of the input and output values (i.e. replacing the values with their ranks) will result in linear relationships and the rank correlation coefficient will indicate the degree of monotonicity between the input and output sample values (IAEA, 1989). The rank correlation coefficient, or Spearman's $\rho$, can be calculated using the equation for Pearson's $r$ with the exception of operating on the rank transformed data (Iman and Conover, 1979).

\subsection{The Partial Correlation COEFFicient}

Strong correlations between input parameters may influence input/output correlations. Partial correlation coefficients (PCC) are calculated to account for correlations among other input variables (Gardner et al., 1980; Gardner et al., 1981; Iman et al., 1981a; Iman and Conover, 1982; Otis, 1983; Downing et al., 1985; Iman and Helton, 1985; Breshears, 1987; Whicker and Kirchner, 1987; Iman and Helton, 1988; IAEA, 1989; Whicker et al., 1990; Iman and Helton, 1991; Helton et al., 1993). Given random variables $X_{1}$ and $X_{2}$ as input and the output variable $Y$, a partial correlation coefficient is a measure of the correlation between $X_{1}$ and $Y$, for example, while eliminating indirect correlations due to relationships that may exist between $X_{1}$ and $X_{2}$ or $X_{2}$ and $Y$. The PCC is defined as (Conover, 1980)

$$
r_{X_{1} Y \mid X_{2}}=\frac{r_{X_{1} Y}-r_{X_{1} X_{2}} r_{X_{2} Y}}{\sqrt{\left(1-r_{X 1 X_{2}}^{2}\right)\left(1-r_{X 2 Y}^{2}\right)}},
$$

where the notation $r_{X_{1} Y \mid X_{2}}$ represents the partial correlation coefficient for $X_{1}$ and $Y$ while accounting for the affects of $X_{2}$. The parameters of the generic model considered in this report are assumed to be independent and no correlations have been assigned, i.e. $r_{X_{1} X_{2}}=0$. Therefore, $r_{X_{1} Y \mid X_{2}}$ reduces to

$$
r_{X_{1} Y \mid X_{2}}=\frac{r_{X_{1} Y}}{\sqrt{\left(1-r_{X_{2} Y}^{2}\right)}},
$$

where, again, $X_{1}$ and $X_{2}$ represent any two input variables and $Y$ represents the output variable. The square of the partial correlation coefficient is useful in determining the percentage of variability in $Y$ accounted for by variability in $X_{i}$ (Gardner 
et al., 1981). Sensitivity rankings based on the relative values of the partial correlation coefficients will not change from the rankings determined based on the simple correlation coefficients. Therefore, with no correlations existing between input parameters, there is no need for calculating partials to determine sensitivity rankings.

The rank transformation can also be applied to partial correlation as a test of monotonicity between input and output variables while accounting for relationships between input parameters. The partial rank correlation coefficient (PRCC) is widely utilized for sensitivity studies (Iman et al., 1981a, b; Crick et al., 1987; Iman and Helton, 1988; IAEA, 1989; Iman and Helton, 1991). Downing et al. (1985) compared parameter sensitivity rankings determined using partial rank correlation with orders from their three importance rankings (see section above on Importance Factors). They report the PRCC to be more powerful at indicating the sensitivity of a parameter that is strongly monotonic yet highly nonlinear.

\subsection{REGRESSION TECHNIQUES}

Regression methods are often used to replace a highly complex model with a simplified 'response surface' (Cox, 1977; Iman et al., 1978; Iman et al., 1981a, b; Helton and Iman, 1982; Downing et al., 1985; Kim et al., 1985; Iman and Helton, 1988; Helton et al., 1991). The response surface is simply a regression equation that approximates model output using only the most sensitive model input parameters. Stepwise regression procedures are utilized to ensure that the final regression model provides for the best fit of raw data (Iman et al., 1978; Iman and Conover, 1980; Iman et al., 1981b; Iman and Conover, 1982; Helton and Iman, 1982; Reed et al., 1984; Helton et al., 1985, 1986; IAEA, 1989; Iman and Helton, 1991; Zimmerman et al., 1991; Helton et al., 1991, 1993). The stepwise regression may involve higher ordered equations, quadratic terms, and parameters as functions of other parameters.

Regression coefficients provide a means of applying sensitivity rankings to input parameters and have been used for such in several investigations (Iman and Conover, 1980; Iman et al., 1981b; Helton et al., 1985; Kim et al., 1985; Helton et al., 1986; Whicker and Kirchner, 1987; Whicker et al., 1990; Margulies et al., 1991; Zimmerman et al., 1991; Kleijnen et al., 1992; Helton et al., 1993). A model with many sensitive parameters may result in a complex regression equation. Matrix techniques have been utilized in such cases to calculate the regression coefficients (Krieger et al., 1977).

The generalized form of a simple regression equation is,

$$
\hat{Y}=b_{0}+\sum_{k} b_{k} Z_{k}
$$

where each $Z_{k}$ is a predictor variable and a function of $\left(X_{1}, \ldots, X_{n}\right)$ and each $b_{k}$ is a regression coefficient (Helton et al., 1985, 1986). The use of the regression 
technique allows the sensitivity ranking to be determined based on the relative magnitude of the regression coefficient. This value is indicative of the amount of influence the parameter has on the whole model. Because of units and the relative magnitudes of parameters, a standardization process is sometimes warranted, however.

\subsection{STANDARDIZED REGRESSION TECHNIQUES}

Standardization takes place in the form of a transformation by ranks or by the ratio of the parameter's standard deviation to its mean. The effect of the standardization is to remove the influence of units and place all parameters on an equal level. Standardized regression analyses are performed by Iman and Helton (Helton et al., 1985; Iman and Helton, 1988, 1991).

The calculation of a rank regression coefficient (RRC), i.e. standardization by the rank transformation, is a simple procedure requiring less computation. Utilizing means and standard deviations of input and output data sets (the standardized regression coefficient, SRC), however, is slightly more rigorous and is achieved by

$$
\frac{(\hat{Y}-\bar{Y})}{s}=\sum_{k}\left[\frac{b_{k} s_{k}}{s}\right] \frac{\left(Z_{k}-\bar{Z}_{k}\right)}{s_{k}},
$$

where each $Z_{k}$ is a function of $\left(X_{1}, \ldots, X_{n}\right), s$ is the standard deviation of the output, and $s_{k}$ is the standard deviation of the input (Helton et al., 1985, 1986). If each $Z_{k}$ is a function of only one parameter in $X$, then the value of $b_{k} s_{k} \mathrm{x} s$ is the standardized regression coefficient for parameter $X_{k}$, where $k=1$ to $n$.

The PRCC estimated in the section above and the standardized regression coefficient are essentially the same when using ranks; the numerical values may be different but both exhibit the same pattern of sensitivity ranking (Iman and Helton, 1988).

\section{Sensitivity Tests Involving Segmented Input Distributions}

These statistical tests involve dividing or segmenting input parameter distributions into two or more empirical distributions based on an associated partitioning of the output (Crick et al., 1987). The tests are utilized to compare the characteristics of the input distributions created by the segmentation. For example, if a dose distribution is calculated and the median value of the distribution is chosen as the dividing point, all input values for the parameter in question associated with the calculation of a dose value below the median are said to belong to one random sample while the input values associated with dose estimates above the median belong to a second random sample. Means, medians, variances, and other characteristics of the independent random samples are statistically compared to determine whether 
the samples originated from the same population. Division of the output distribution can occur at any value or percentile, but should be based on the statistical question to be answered, e.g. 'Is the model more sensitive to the parameter when determining the mean value or when estimating maximum values'. If the input distributions generated by this process are statistically identical then the model is not sensitive to that parameter. However, if the distributions are different then the output distribution is indeed influenced by the input and the absolute value of the test statistic can be used to perform the sensitivity ranking.

Standard parametric tests are not reasonable on input data sets generated by random sampling methods because of our limited knowledge of the input variables and their associated distributions (Iman et al., 1981b). Nonparametric statistical tests, therefore, are used where the data are considered to be distribution-free (Conover, 1980). The four nonparametric statistical tests that follow (Smirnov, Cramer-von Mises, Mann-Whitney, and Squared Ranks) are used to determine whether the null hypothesis can be accepted. The Smirnov and Cramer-von Mises tests compare empirical distributions with a null hypothesis of 'the distributions originate from the same population'. The Mann-Whitney test and the Squared Ranks test compare means and variances, respectively, of the empirical distributions. These test statistics are calculated for the purpose of sensitivity ranking, however, and not for accepting or rejecting null hypotheses.

The convention stated earlier, that $Y$ is a function of $X\left(Y=f\left\{X_{1}, \ldots, X_{n}\right\}\right)$, is no longer appropriate; a new notation is used and specified for each test. The following tests operate on ranks of the raw data. Tied values are assumed not to exist because the input and output values can be determined to several significant figures (although this feature does not necessarily reflect a high degree of precision). By excluding the possibility of ties, equations for calculating the test statistics are greatly simplified (Conover, 1980).

\subsection{The SMIRnov TeST}

The Smirnov test operates on the two empirical distributions, $S_{1}(X)$ and $S_{2}(x)$, generated as a result of partitioning the input parameter values. The degree of similarity between distributions, measured by the test statistic, is used to indicate the degree of sensivity between the input and output values.

The Smirnov test statistic can be measured directly as the greatest vertical distance between the two distribution functions plotted on the same graph (see Figure 1), or the test statistic can be calculated using,

$$
T_{1}=\sup \left|S_{1}(x)-S_{2}(x)\right|,
$$

where 'sup' is the abbreviation for supremum and the equation represents the greatest absolute difference between $S_{1}(x)$ and $S_{2}(x)$ (Conover, 1980). In the example of Figure 1, a plot is shown of two empirical distributions of input values resulting in an output less than the median and input values resulting in an output 


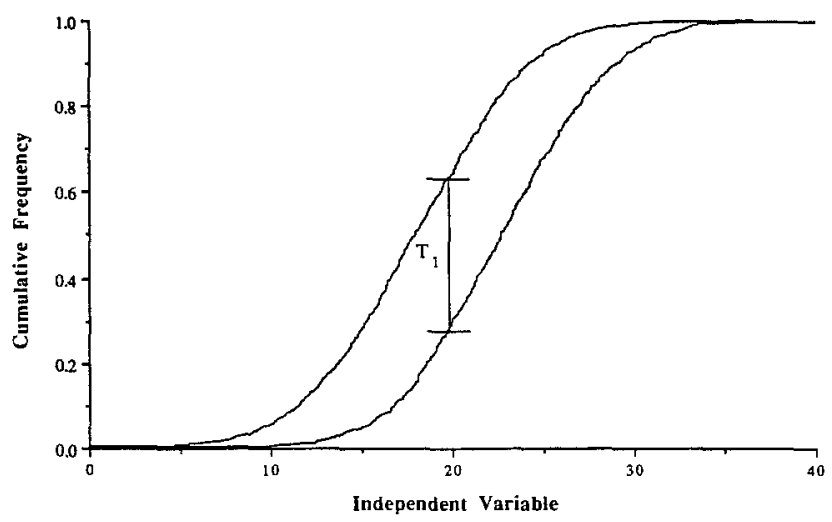

Fig. 1. Example of the graphical determination of the Smirnov statistic. The value of $T_{1}$ is a measure of the disparity of the two empirical distributions.

greater than the median. The value of the Smirnov statistic can be taken from the plot or its accuracy can be increased by calculating $T_{1}$ from the two empirical distributions.

\subsection{The Cramer-von Mises Test}

The Cramer-von Mises test is very similar to the Smirnov test in that its purpose is to determine whether two empirical distributions are statistically identical. The computation of the test statistic is slightly more complicated, yet there is little difference in the test's power compared to the Smirnov statistic (Conover, 1980). The Cramer-von Mises statistic, $T_{2}$, is the sum of all squared vertical distances between the two empirical distributions,

$$
T_{2}=\frac{m n}{(M+n)^{2}} \sum\left[S_{1}(x)-S_{2}(x)\right]^{2},
$$

where the values of $n$ and $m$ are the number of samples utilized to estimate the distributions. It is expected that the parameter rankings based on the Smirnov and Cramer-von Mises tests will be very similar since the two tests show little difference in their statistical power.

Recall that a large Smirnov or Cramer-von Mises statistic is indicative of a larger difference in the two empirical distributions generated by the division of input data based on some output criteria. This large difference indicates a greater correlation between the independent and dependent variables.

\subsection{The MAnN-Whitney Test}

The Mann-Whitney test, also known as the Wilcoxon test, is utilized to compare the means of two independent samples (Conover, 1980). Two distribution functions, $X$ and $Y$, are ordered as a single sample and ranks are assigned based on the ordering; 
ties are assumed not to exist. The test statistic, $T$, is the sum of the resulting ranks of the data from distribution $X$,

$$
T=\sum_{i=1}^{n} R\left(X_{i}\right),
$$

where $R\left(X_{i}\right)$ refers to the rank of $X_{i}$. In theory, if one sum of the single-sample ranks is much larger than the other, the two sample means are different (Conover, 1980 ), i.e., the test statistic is either very small or very large. The use of ranks in the Mann-Whitney test is preferred over the use of raw data since probability theory does not depend on distribution-type when operating on ranks (Conover, 1980).

For hypothesis testing, a range is calculated in order to accept or reject the null hypothesis that the two distributions have the same mean value. A calculated test statistic larger than the midpoint indicates that the mean value of the first empirical distribution is larger than the mean value of the second. Since the Mann-Whitney test is two-tailed, sensitivity ranks are based on an adjusted value of $T$. The adjusted statistic is either the value of the test statistic itself, if it is less than the midpoint of the comparison range, or the test statistic minus twice the difference between $T$ and the midpoint, if it is greater than the midpoint. For example, if the midpoint of the comparison range is 500 and a value of 400 is calculated for $T$, the value of $T$ is not adjusted. If, however, the calculated $T$ is 550 , the adjusted value becomes 450. Then, smaller values of $T$ indicate the more sensitive parameters since the means of the distributions show a greater difference based on the partitioning of input data.

\subsection{The SQuared-Ranks Test}

The variances of two independent samples, $X_{i}$ and $Y$, can be compared using the squared-ranks test. Ranks are not based on the raw data, rather on the absolute difference between the random sample (e.g. $X_{i}$ ) and the sample mean (e.g. $\mu_{x}$ ). There are assumed to be no ties and the ranks are assigned to a single sample of the two distributions based on this transformation. The ranks then are squared, to provide more statistical power (Conover, 1980), and summed in a fashion similar to the Mann-Whitney test. The test statistic, $T$, is equal to

$$
T=\sum_{i=1}^{n}\left[R\left(U_{i}\right)\right]^{2},
$$

where

$$
U_{i}=\left|X_{i}-\mu_{x}\right| .
$$

For parameter ranking purposes, the normalization procedure executed on the Mann-Whitney statistic also is necessary with the squared-ranks statistic. 
Based on preliminary numerical comparisons to other sensitivity tests, the squared-ranks test does not appear to be of much value for parameter sensitivity rankings. Uncertainty analyses may benefit, however, in that the amount of variability in the output distribution may be shown to be influenced by input values that result in either high or low output values.

\section{Summary and Discussion}

A number of sensitivity analysis techniques have been presented. The majority of investigators utilize only a few of the methods including partial differentiation, partial correlation, and regression techniques. Investigators report on sensitivity theory and techniques related to environmental transport, reactor safety, chemical kinetic models, radiation dosimetry, multi-compartment ecological models, and general mathematical and statistical theory.

The simplest approach to sensitivity analysis is the one-at-a-time method where sensitivity measures are determined by varying each parameter while all others are held constant. The factorial design is easy to conceptualize, but its procedure can become quite intensive with larger models. Standardized regression analysis using a stepwise regression procedure appears to be the most comprehensive technique and is relatively easy to perform with commercially available software. If possible, complex models should be carried through an aggregating process in the screening stage to simplify the model's structure and reduce the number of input parameters.

The most fundamental of sensitivity techniques is the direct method. Direct sensitivity analysis involves calculating partial derivatives of the model with respect to each input parameter. The technique is only valid for small variability in parameter values and the partials must be recalculated for each change in the base-case scenario (e.g. all parameters set to their mean value). As stated above, 'local' sensitivity methods, or one-at-a-time analyses, are conceptually the simplest methods. These too are valid only for the base-case. When working with complex models a subjective analysis, whereby experienced modelers discard unimportant variables, may be necessary as a screening tool to reduce the number of parameters to a manageable size.

Certain sensitivity analyses examine parameter influence based on output variation while simultaneously changing input parameters. These methods are easily performed by utilizing a random sampling technique to build an input matrix and then calculating an output vector. Correlation, rank correlation, and partial rank correlation coefficients can be calculated to determine relationships between the independent and dependent variables. Rank transformations are often utilized in correlation analysis to eliminate the forced assumption of linear correlations among the raw data. Investigators typically calculate partial correlations to eliminate influences between two or more input parameters. Regression analysis is commonly 
utilized to investigate parameter sensitivity and to build response surfaces that approximate complex models. Standardized regression coefficients are used as sensitivity measures since the standardization process removes the effect of units and places all parameters on a comparable level.

Crick et al. (1987) propose statistically analyzing selected input vectors by segmenting input values based on their relationship to some critical output value (e.g. the mean, median, or 90th percentile of the output distribution). This type of analysis provides detailed information on parameter sensitivity based on the calculated output. Nonparametric statistical tests are used to determine whether the inputs contributing to an output less than the critical value are any different than the inputs contributing to an output greater than the critical value. For example, the analysis may show that a model is much more sensitive to a particular input when the output is greater than its mean value. The nonparametric tests on partitioned data sets are labor intensive and are not necessarily beneficial unless a particular question is to be answered regarding the sensitivity of a parameter with respect to the range of the model output.

Generally, the purpose of a sensitivity analyses is to determine which input parameters exert the most influence on model results. This information, in turn, allows for unimportant parameters to be eliminated and provides direction for further research in order to reduce parameter uncertainties and increase model accuracy. Strict adherence to sensitivity rankings, therefore, is not as important as is the determination of the top several parameters to which the model is most sensitive. While some sensitivity methods are mathematically elegant and comprehensive, their use is inefficient and their results, in many cases, are comparable to those obtained from simpler techniques. Since very few comprehensive studies exist that indicate the superiority of one approach over several others (Rose, 1983; Dalrymple and Broyd, 1987), a study was completed to compare the methods numerically and provide recommendations on their use (Hamby, 1995).

\section{Acknowledgements}

The author would like to thank Drs. L.R. Bauer and W.H. Carlton for their critical reviews of this manuscript. This work was supported by the U.S. Department of Energy under Contract No. DE-AC09-89SR 18035 with the Westinghouse Savannah River Company.

\section{References}

Atherton, R.W., Schainker, R.B., and Ducot, E.R.: 1975, 'On the Statistical Sensitivity Analysis of Models for Chemical Kinetics', AlChE. 21, 441-448.

Bauer, L.R., and Hamby, D.M.: 1991, 'Relative Sensitivities of Existing and Novel Model Parameters in Atmospheric Tritium Dose Estimates', Rad. Prot. Dosimetry. 37, 253-260.

Bellman, R., and Astrom, K.J.: 1970, 'On Structural Identifiability', Math. Biosci. 7, 329-339. 
Box, G.E.P., Hunter, W.G., and Hunter, J.S.: 1978, Statistics for Experimenters: an Introduction to Design, Data Analysis, and Model Building. John Wiley \& Sons. New York.

Breshears, D.D.: 1987, Uncertainty and sensitivity analyses of simulated concentrations of radionuclides in milk. Fort Collins, CO: Colorado State University, MS Thesis, pp. 1-69.

Cacuci, D.G., Weber, C.F., Oblow, E.M., and Marable, J.H.: 1980. 'Sensitivity Theory for General systems of Nonlinear Equations', Nuc. Sci. and Eng. 75, 88-110.

Cacuci, D.G., Maudlin, P.J., and Parks, C.V.: 1983, 'Adjoint Sensitivity Analysis of Extremum-Type Responses in Reactor Safety,' Nuc. Sci. and Eng. 83, 112-135.

Cobelli, C., and Romanin-Jacur, G.: 1976, 'Controllability, Observability and Structural Identifiability of Multi Input and Multi Output Biological Compartmental Systems', IEEE Trans. Biomed. Eng. 23, 93.

Cobelli, C., and DiStefano, J.J.: 1980, 'Parameter and Structural Identifiability Concepts and Ambiguities: A critical Review and Analysis', Amer. J. Physiol. 239, R7-R24.

Conover, W.J.: 1980, Practical Nonparametric Statistics. 2nd edn. John Wiley \& Sons, New York. Cox, N.D.: 1977, 'Comparison of Two Uncertainty Analysis Methods', Nuc. Sci. and Eng. 64, 258-265.

Crick, M.J., Hill, M.D. and Charles, D.: 1987, 'The Role of Sensitivity Analysis in Assessing Uncertainty. In: Proceedings of an NEA Workshop on Uncertainty Analysis for Performance Assessments of Radioactive Waste Disposal Systems, Paris, OECD, pp. 1-258.

Cukier, R.I., Fortuin, C.M., Shuler, K.E., Petschek, A.G. and Schaibly, J.H.: 1973, 'Study of the Sensitivity of Coupled Reaction Systems to Uncertainties in Rate Coefficients. I. Theory $J$. Chem. Phys. 59, 3873-3878.

Cukier, R.I., Levine, H.B. and Schuler, K.E.: 1978, 'Nonlinear Sensitivity Analysis of Multiparameter Model Systems', J. Computational Phys. 26, 1-42.

Cukier, R.I., Schaibly, J.H. and Shuler, K.E.: 1975, 'Study of the Sensitivity of Coupled Reaction Systems to Uncertainties in Rate Coefficients. III. Analysis of the Approximations', J. Chem. Phys. 63, 1140-1149.

Cunningham, M.E., Hann, C.R., and Olsen, A.R.: 1980, 'Uncertainty Analysis and Thermal Stored Energy Calculations in Nuclear Fuel Rods', Nuc. Technol. 47, 457-467.

Dalrymple, G.J., and Broyd, T.W.: 1987, 'The Development and Use of Parametric Sampling Techniques for Probabilistic Risk Assessment. IAEA-SR-111/54P', In: Implications of Probabilistic Risk Assessment (M.C. Cullingford, S.M. Shah, and J.H. Gittus eds.), Elsevier, London, pp. 171186.

Demiralp, M., and Rabitz, H.: 1981, 'Chemical Kinetic Functional Sensitivity Analysis: Elementary Sensitivities', J. Chem. Phys. 74, 3362-3375.

Dickinson R.P., and Gelinas, R.J.: 1976, 'Sensitivity Analysis of Ordinary Differential Equation Systems - A Direct Method', J. Computational Phys. 21, 123-143.

Downing, D.J., Gardner, R.H., and Hoffman, F.O.: 1985, 'An Examination of Response-Surface Methodologies for Uncertainty Analysis in Assessment Models', Technometrics. 27, 151-163. (See also Letter to the Editor, by R.G. Easterling and a rebuttal, Technometrics 28, 91-93, 1986.)

Dunker, A.M.: 1981, 'Efficient Calculation of Sensitivity Coefficients for Complex Atmospheric Models', Atmospheric Environment 15, 1155-1161.

Gardner, R.H.: Huff, D.D., O'Neill, R.V., Mankin, J.B., Carney, J. and Jones, J.: 1980, 'Application of Error Analysis to a Marsh Hydrology Model', Water Resources Res. 16, 659-664.

Gardner, R.H., O'Neill, R.V., Mankin, J.B. and Carney, J.H.: 1981, 'A Comparison of Sensitivity Analysis and Error Analysis Based on a Stream Ecosystem Model', Ecol. Modelling. 12, 173190.

Hall, M.C.G., Cacuci, D.G., Schlesinger, M.E.: 1982, 'Sensitivity Analysis of a Radiative-Convective Model by the Adjoint Method', J. Atmos. Sci. 39, 2038-2050.

Hamby, D.M.: 1993, 'A Probabilistic Estimation of Atmospheric tritium Dose', Health Phys. 65, $33-40$.

Hamby, D.M. 1995, ‘A Numerical Comparison of Sensitivity Analysis Techniques', scheduled to appear in Health Phys. 68. 
Helton, J.C., Garner, J.W., Marietta, M.G., Rechard, R.P., Rudeen, D.K. and Swift, P.N.: 1993. 'Uncertainty and Sensitivity Analysis Results Obtained in a Preliminary Performance assessment for the Waste Isolation Pilot Plant, Nuc. Sci. and Eng. 114, 286-331.

Helton, J.C., Garner, J.W., McCurley, R.D. and Rudeen, D.K.: 1991, Sensitivity analysis techniques and results for performance assessment at the waste isolation pilot plant. Albuquerque, NM: Sandia National Laboratory; Report No. SAND90-7103.

Helton, J.C. and Iman, R.L.: 1982, 'Sensitivity Analysis of a Model for the Environmental Movement of Radionuclides', Health Phys. 42, 565-584.

Helton, J.C., Iman, R.L. and Brown, J.B.: 1985, 'Sensitivity Analysis of the Asymptotic Behavior of a Model for the Environmental Movement of Radionuclides, Ecol. Modelling. 28, 243-278.

Helton, J.C., Iman, R.L., Johnson, J.D., and Leigh, C.D.: 1986, 'Uncertainty and Sensitivity Analysis of a Model for Multicomponent Aerosol Dynamics, Nuc. Technol. 73, 320-342.

Hoffman, F.O. and Gardner, R.H.: 1983, 'Evaluation of Uncertainties in Environmental Radiological Assessment Models', in: Till, J.E.; Meyer, H.R. (eds) Radiological Assessments: a Textbook on Environmental Dose Assessment. Washington, DC: U.S. Nuclear Regulatory Commission; Report No. NUREG/CR-3332.

Horwedel, J.E, Worley, B.A., Oblow, E.M., Pin, F.G. and Wright, R.Q.: 1988, GRESS version 0.0 user's manual. Oak Ridge, TN: Oak Ridge National Laboratory; Report No. ORNL/TM-10835.

Iman, R.L.: 1987, 'A Matrix-Based Approach to Uncertainty and Sensitivity Analysis for Fault Trees', Risk Analysis. 7, 21-33.

Iman, R.L. and Conover, W.J.: 1979, 'The Use of the Rank Transform in Regression', Technometrics. 21, 499-509.

Iman, R.L. and Conover, W.J.: 1980 'Small Sample Sensitivity Analysis Techniques for Computer Models, With an Application to Risk Assessment', Commun. Stats. - Theor. and Meth. A9, 1749-1842.

Iman, R.L. and Conover, W.J.: 1982, Sensitivity-analysis techniques: self-teaching curriculum. Albuquerque, NM: Sandia National Laboratories; Report No. NUREG/CR-2350.

Iman, R.L. and Helton, J.C.: 1985, A comparison of uncertainty and sensitivity analysis techniques for computer models. Albuquerque, NM: Sandia National Laboratory; Report No. NUREG/CR3904.

Iman, R.L., and Helton, J.C. 1988, 'An Investigation of Uncertainty and Sensitivity Analysis Techniques for Computer Models', Risk Analysis. 8, 71-90.

Iman, R.L., and Helton, J.C., 1991; 'The Repeatability of Uncertainty and Sensitivity Analyses for Complex Probabilistic Risk Assessments', Risk Analysis. 11, 591-606.

Iman, R.L. Helton, J.C. and Campbell, J.E. 1978, Risk methodology for geologic disposal of radioactive waste: sensitivity analysis techniques. Albuquerque, NM: Sandia National Laboratories; Report No. NUREG/CR-0390.

Iman, R.L. Helton, J.C. and Campbell, J.E.: 1981a, 'An Approach to Sensitivity Analysis of Computer Models: Part I - Introduction, Input Variable Selection and Preliminary Assessment', J. Qual. Technol., 13, 174-183.

Iman, R.L. Helton, J.C. and Campbell, J.E.: 1981b, 'An Approach to sensitivity Analysis of Computer Models: Part II - Ranking of Input Variables, Response Surface Validation, Distribution Effect and Technique Synopsis', J. Qual. Technol. 13, 232-240.

Iman, R.L. and Shortencarier, M.J.: 1984, A FORTRAN 77 program and user's guide for the generation of Latin hypercube and random samples for use with computer models. Albuquerque, NM: Sandia National Laboratory; Report No. NUREG/CR-3624.

International Atomic Energy Agency (IAEA), '1989, Evaluating the reliability of predictions made using environmental transfer models. Vienna: Safety Series No. 100. Report No. STI/PUB/835; $1-106$.

Kim, T.W., Chang, S.H. and Lee, B.H., 1988 'Uncertainty and Sensitivity Analyses in Evaluating Risk of High Level Waste Repository', Rad. Waste Management and the Nuc. Fuel Cycle. 10, 321-356.

Kleijnen, J.P.C., van Ham, G., and Rotmans, J., 1992, 'Techniques for Sensitivity Analysis of Simulation Models: A Case Study of the $\mathrm{CO}_{2}$ Greenhouse Effect', Simulation. 58, 410 . 
Koda, M., Dogru, A.H., Seinfeld, and J.H.: 1979 'Sensitivity Analysis of Partial Differential Equations with Application to Reaction and Diffusion Processes'. J. Computational Phys. 30, 259-282. Koda, M. 1982, 'Sensitivity Analysis of the Atmospheric Diffusion Equation', Atmos. Environment, 16, 2595-2601.

Krieger, T.J., Durston, C., and Albright, D.C.: 1977 Statistical Determination of Effective Variables in Sensitivity Analysis', Trans. Am. Nuc. Soc. 28, 515-516.

Liepmann, D., and Stephanopoulos, G., 1985, 'Development and Global Sensitivity Analysis of a Closed Ecosystem Model,' Ecol. Modelling. 30, 13-47.

Margulies, T., Lancaster, L., and Kornasiewicz, R.A.: 1991, 'Uncertainty and Sensitivity Analysis of Environmental Transport Models for Risk Assessment', In: Engineering Applications of Risk Analysis, Atlanta, GA: ASME, 11-19.

McKay, M.D., Beckman, R.J. and Conover, W.J.: 1979, 'A Comparison of Three Methods for Selecting Values of Input Variables in the Analysis of Output from a Computer Code, Technometrics. 21, 239-245.

Morisawa, S., and Inoue, Y. 1974, 'On the Selection of a Ground Disposal Site by Sensitivity Analysis', Health Phys. 26, 251-261.

Oblow, E.M.: 1978, 'Sensitivity Theory for General Nonlinear Algebraic Equations with Constraints', Nuc. Sci. and Eng. 65, 187-191.

O'Neill, R.V., Gardner, R.H., and Mankin, J.B. 1980, 'Analysis of Parameter Error in a Nonlinear Model', Ecol. Modelling. 8, 297-311.

Otis, M.D.: 1983, 'Sensitivity and Uncertainty Analysis of the PATHWAY Radionuclide Transport Model', Fort Collins, CO: Colorado State University, PhD Dissertation, pp. 1-86.

Reed, K.L., Rose, K.A., and Whitmore, R.C. 1984, 'Latin Hypercube Analysis of Parameter Sensitivity in a Large Model of Outdoor Recreation Demand, Ecol. Modelling. 24, 159-169.

Rose, K.A.: 1983, 'A Simulation Comparison and Evaluation of Parameter Sensitivity Methods Applicable to Large Models, in: Analysis of Ecological Systems: State-of-the-art in Ecological Modelling (Lauenroth, G.V. Skogerboe, M. Flug eds.), Proceedings of a meeting at Colorado State University, May 24-28, 1982 Elsevier, New York.

Schaibly, J.H., and Shuler, K.E. 1973, 'Study of the sensitivity of coupled reaction systems to uncertainties in rate coefficients. II. Applications', J. Chem. Phys. 59, 3879-3888.

Stein, M.: 1987, 'Large Sample Properties of Simulations Using Latin Hypercube Sampling', Technometrics. 29, 143-151.

Summers, J.K., and McKellar, H.N.: 1981, 'A sensitivity analysis of an Ecosystem Model of Estuarine Carbon Flow,' Ecol. Modelling. 13; 283-301.

Whicker, F.W., and Kirchner, T.B.: 1987, 'PATHWAY: A dynamic food-chain model to predict radionuclide ingestion after fallout deposition,' Health Phys. 52; 717-737.

Whicker, F.W., Kirchner, T.B., Breshears, D.D., and Otis, M.D. 1990, 'Estimation of radionuclide ingestion: the "Pathway" model', Health Phys. 59; 645-657.

Worley, B.A., and Horwedel, J.E.: 1986. A waste package performance assessment code with automated sensitivity-calculation capability. Oak Ridge, TN: Oak Ridge National Laboratory; Report No. ORNL/TM-9976.

Yu, C., Cheng, J-J., and Zielen, A-J.: 1991, 'Sensitivity Analysis of the RESRAD, a Dose Assessment Code,' Trans. Am. Nuc. Soc. 64; 73-74.

Zirnmerman, D.A., Hanson, R.T., and Davis, P.A.: 1991, A comparison of parameter estimation and sensitivity analysis techniques and their impaet on the uneertainty in ground water flow model predictions. Albuquerque, NM: Sandia National Laboratory; Report No. NUREG/CR-5522. 